\title{
Neural Machine Translation with Byte-Level Subwords
}

\author{
Changhan Wang, ${ }^{\dagger}$ Kyunghyun Cho ${ }^{\dagger \ddagger \star}$ Jiatao $\mathrm{Gu}^{\dagger}$ \\ ${ }^{\dagger}$ Facebook AI Research; ${ }^{\ddagger}$ New York University; ${ }^{\star}$ CIFAR Global Scholar \\ \{changhan, kyunghyuncho, jgu\}@fb.com
}

\begin{abstract}
Almost all existing machine translation models are built on top of character-based vocabularies: characters, subwords or words. Rare characters from noisy text or character-rich languages such as Japanese and Chinese however can unnecessarily take up vocabulary slots and limit its compactness. Representing text at the level of bytes and using the 256 byte set as vocabulary is a potential solution to this issue. High computational cost has however prevented it from being widely deployed or used in practice. In this paper, we investigate byte-level subwords, specifically byte-level BPE (BBPE), which is compacter than character vocabulary and has no out-of-vocabulary tokens, but is more efficient than using pure bytes only is. We claim that contextualizing BBPE embeddings is necessary, which can be implemented by a convolutional or recurrent layer. Our experiments show that BBPE has comparable performance to BPE while its size is only $1 / 8$ of that for BPE. In the multilingual setting, BBPE maximizes vocabulary sharing across many languages and achieves better translation quality. Moreover, we show that BBPE enables transferring models between languages with non-overlapping character sets.
\end{abstract}

\section{Introduction}

It has become a standard practice to build a vocabulary in neural machine translation (NMT) (Bahdanau, Cho, and Bengio 2014; Sutskever, Vinyals, and Le 2014) using bytepair encoding (BPE) (Sennrich, Haddow, and Birch 2015). In this practice, we notice that BPE is used at the level of characters rather than at the level of bytes, which is more common in data compression. We suspect this is because text is often represented naturally as a sequence of characters, although it has recently been noticed that byte representation of text has its own advantages, such as compactness (up to 256 possible values) and being agnostic to languages.

In this paper, we look into byte-level "subwords" that are used to tokenize text into variable-length byte $n$-grams, as opposed to character-level subwords in which we represent text as a sequence of character $n$-grams. We specifically focus on byte-level BPE (BBPE), examining compact BBPE vocabularies in both bilingual and multilingual settings as

Copyright (C) 2020, Association for the Advancement of Artificial Intelligence (www.aaai.org). All rights reserved.

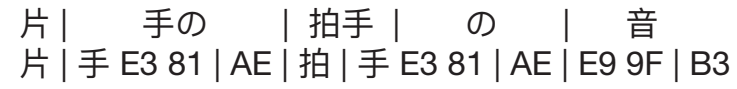

Figure 1: BPE (upper) and BBPE (lower) tokenization of a Japanese sentence. Bytes (from partial characters) are represented by hexadecimal digits.

well as in a novel setup of transfer learning to a new language with a non-overlapping character set.

\section{Byte Level Text Representation}

Encoding Byte-Level Representation We consider UTF8 encoding of text, which encodes each Unicode character into 1 to 4 bytes. This allows us to model a sentence as a sequence of bytes instead of characters. While there are $138 \mathrm{~K}$ Unicode characters covering over 150 languages, we represent a sentence in any language as a sequence of UTF-8 bytes ( 248 out of 256 possible bytes).

A byte sequence representation of text is often much longer (up to $4 \mathrm{x}$ ) than a character sequence representation, which makes it computationally demanding to use bytes as they are. As an alternative, we consider segmenting a byte sequence into variable-length $n$-grams (byte-level "subwords"). Specifically, we learn BPE vocabulary on the byte-level representation which extends UTF- 8 byte set with byte $n$-grams. We denote this type of vocabulary as $\mathrm{B}$ (ytelevel)BPE in the rest of the paper. Figure 1 shows an example of BBPE tokenization.

BBPE symbols can be partial characters shared by different characters or the combination of complete and partial characters. This arbitrariness may necessitate incorporating a larger context surrounding each symbol for disambiguation and learning the character boundaries. In this work, we base our experiments on Transformer (Vaswani et al. 2017) models. We propose to use either a depth-wise convolutional layer (Kaiser, Gomez, and Chollet 2017) or a bidirectional recurrent layer with gated recurrent units (Cho et al. 2014, GRU,) to contextualize BBPE embeddings before feeding them into the model: 


$$
\mathbf{x}_{c t x_{-} e m b}=\operatorname{DepthWiseConv}\left(\mathbf{x}_{e m b}\right)
$$

or

$$
\mathbf{x}_{c t x \_e m b}=\operatorname{BiGRU}\left(\mathbf{x}_{e m b}\right)
$$

Decoding with Byte-Level Subwords While any sentence can be represented as a byte sequence, the converse is, however, not necessarily true in that there are byte sequences that do not translate to valid character sequences. Empirically, we find that invalid outputs from trained models are very rare. We do not observe any in the experiments described below (note that one of them does have a large test set of $165 \mathrm{~K}$ examples). And a common error pattern in halftrained models is redundant repeating bytes. In our system, we try to recover as many Unicode characters as possible from this error pattern efficiently in linear time. The algorithm is as follows: For a given byte sequence $\{B\}_{k=1}^{N}$, we denote the maximum number of characters that we can recover from it as $f(k)$. Then $f(k)$ has optimal substructure and can be solved by dynamic programming:

$$
f(k)=\max _{t=1,2,3,4}\{f(k-t)+g(k-t+1, k)\}
$$

where $g(i, j)=1$ if $\{B\}_{k=i}^{j}$ corresponds to a valid character, otherwise 0 . When $f(k)$ is calculated recursively, we also record the selections at each position $k$ so that we can recover the solution through backtracking. The design of UTF-8 encoding ensures the uniqueness of this recovery process: for a character UTF-8 encoded with multiple bytes, its trailing bytes will not make a valid UTF-8 encoded character. Then the best selection in Eq. 1 is unique and so is the final solution.

\section{Experimental Settings}

Datasets We run experiments on three bilingual corpora as well as a many-to-English multilingual dataset:

- English-German (En-De): we replicate the same setting of (Vaswani et al. 2017) which uses WMT $2014^{1}$ data (newstest13 for validation and newstest14 for testing)

- Japanese-English (Ja-En): we follow (Michel and Neubig 2018) and concatenate $\mathrm{KFTT}^{2}$ (Neubig 2011), TED $^{3}$ (Cettolo, Girardi, and Federico 2012) and JESC $^{4}$ (Pryzant et al. 2017) to construct training, validation and test sets.

- Sinhala-English (Si-En): we use the data from FLoRes (Guzmán et al. 2019).

- Many-to-English (X-En): we adopt the TED Talks corpus complied by (Ye et al. 2018), which includes parallel data for 59 languages. For our experiments, we use English as target and the other 58 languages as source. We sample $22 \mathrm{~K}$ examples from the $135 \mathrm{~K}$ development set for validation.

\footnotetext{
${ }^{1} \mathrm{http}: / /$ statmt.org/wmt14/translation-task.html

${ }^{2} \mathrm{http} / / / \mathrm{www}$.phontron.com/kftt

${ }^{3}$ https://wit3.fbk.eu/mt.php?release=2017-01-trnted

${ }^{4}$ https://nlp.stanford.edu/projects/jesc
}

Table 1 shows an overview statistics of these datasets. We learn (B)BPE vocabularies jointly on source and target sentences using SentencePiece (Kudo and Richardson 2018).

\begin{tabular}{c|cccc} 
& En-De & Ja-En & Si-En & X-En \\
\hline Train & $4.5 \mathrm{M}$ & $3.5 \mathrm{M}$ & $405 \mathrm{~K}$ & $5.1 \mathrm{M}$ \\
Dev & $3 \mathrm{~K}$ & $4 \mathrm{~K}$ & $3 \mathrm{~K}$ & $22 \mathrm{~K}^{\star}$ \\
Test & $3 \mathrm{~K}$ & $12 \mathrm{~K}$ & $3 \mathrm{~K}$ & $165 \mathrm{~K}$
\end{tabular}

Table 1: Dataset statistics in number of sentences. $\star$ Subsampled from the full $135 \mathrm{~K}$ development set.

\begin{tabular}{c|cccccc} 
Model & $N$ & $d_{\text {model }}$ & $d_{f f}$ & $h$ & $P_{\text {drop }}$ & Params \\
\hline$T_{\text {flores }}$ & 5 & 512 & 2048 & 2 & 0.4 & $38 \mathrm{M}$ \\
$T_{\text {base }}$ & 6 & 512 & 2048 & 8 & 0.1 & $44 \mathrm{M}$ \\
$T_{\text {big }}$ & 6 & 1024 & 4096 & 16 & 0.3 & $180 \mathrm{M}$
\end{tabular}

Table 2: Transformer models used in the experiments (using the notations in Vaswani et al. 2017).

Models and Learning We use Fairseq (Ott et al. 2019) to train Transformers (Vaswani et al. 2017) with the same learning rate schedule in the original paper. All model configurations are listed in table 2. We set attention and ReLU dropout to 0.1, except $\mathrm{Si}-\mathrm{En}$ for which we use 0.2. We use 0.2 residual dropout for $T_{\text {base }}$ models in X-En. We use a kernel size of 5 and a padding of 2 on both sides for all convolutional layers.

Inference and Evaluation We set beam width to 4 for EnDe and 5 for the other and use the best checkpoint by validation loss to generate the predictions. We calculate casesensitive tokenized BLEU (Papineni et al. 2002) as the metrics using sacreBLEU (Post 2018).

\section{Results and Analysis Qualitative Comparison: BPE vs. BBPE}

Symbol Frequency Distribution Since the construction of BBPE vocabulary starts from UTF-8 byte set, it has the flexibility of decomposing rare characters into byte $n$-grams from the vocabulary instead of including them directly. This frees vocabulary slots for other frequent symbols. Figure 2 compares the symbol frequency distribution of BPE and BBPE. We can see that BBPE symbols are more evenly distributed than BPE ones, even when the latter has already been much more evenly distributed than pure characters. By setting different BBPE vocabulary sizes, we can control the level of rare character decomposition and symbol sharing across different characters. Table 3 shows the ratio of BBPE tokens with partial characters. We can see that large portion of rare characters are decomposed on Ja-En and XEn, which has a large character set of $8 \mathrm{~K}$ and $11 \mathrm{~K}$, respectively. Figure 5 provides an example from Ja-En tokenized with different BBPE vocabularies, where we can see how tokens look like as the tokenization granularity goes from fine to coarse. 

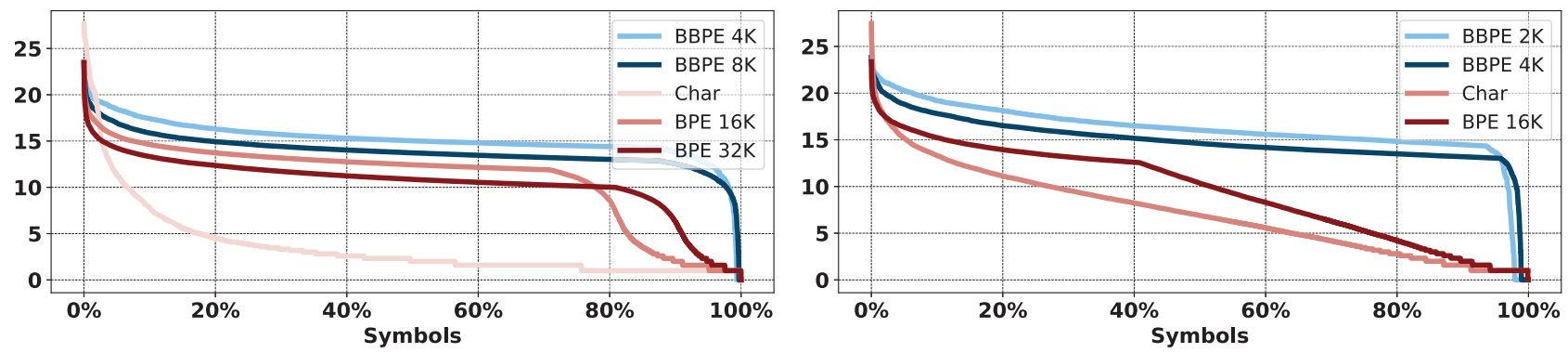

Figure 2: Symbol frequencies (in log2 scale) for En-De (left) and X-En (right) vocabularies. BBPE enables a more consistent distribution of vocabulary across frequencies.

\begin{tabular}{c|ccccc} 
BBPE & $2 \mathrm{~K}$ & $4 \mathrm{~K}$ & $8 \mathrm{~K}$ & $16 \mathrm{~K}$ & $32 \mathrm{~K}$ \\
\hline En-De & $4.3 \%$ & $4.9 \%$ & $5.5 \%$ & $6.1 \%$ & $6.5 \%$ \\
Ja-En & $46.0 \%$ & $47.6 \%$ & $49.4 \%$ & $51.2 \%$ & $34.8 \%$ \\
X-En & $36.8 \%$ & $39.1 \%$ & $41.3 \%$ & $43.6 \%$ & $23.0 \%$
\end{tabular}

Table 3: Ratio of BBPE tokens with partial characters.

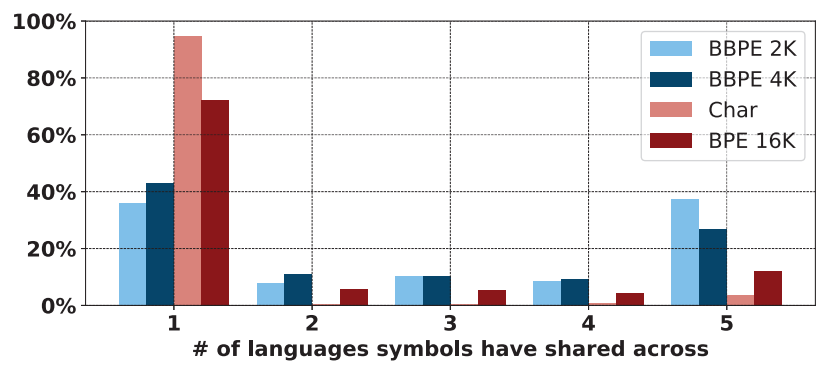

Figure 3: The numbers of languages symbols have shared across $\mathrm{Ar}, \mathrm{He}, \mathrm{Ru}, \mathrm{Ko}$ and It (from X-En). Note that these languages have mutually different character sets.

Cross-Lingual Sharing In the multilingual setting, symbol sharing also happens across different languages despite the different writing systems. This allows maximizing parameter sharing not only for the model part but also the vocabulary part in a universal model. Figure 3 illustrates the level of BBPE symbol sharing across the top 5 languages (by number of train examples) in X-En whose writing systems are different from each other.

Impact on Sequence Lengths Compared to BPE, BBPE symbols are generally finer-grained with shorter byte-level lengths, which results in longer tokenized sequences as well as longer training and inference time. BBPE, however, is optimized for compression-based objective (the same as BPE), and is still more efficient than character vocabulary. Table 4 lists the average lengths of training sentences tokenized with different vocabularies. We can observe that sentences tokenized with BBPE have significantly shorter lengths than the character ones, even when the BBPE vocabulary is much smaller (for example only $1 / 5$ of character set size on XEn). Another observation is that source-target length ratio

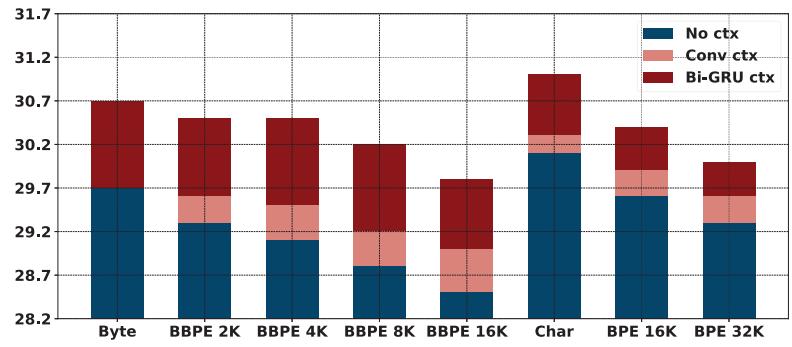

Figure 4: X-En validation BLEU for models without contextualization, with local contextualization (depth-wise convolution) and with long-range contextualization (Bi-GRU). The y axis starts from 28.2 to focus on the gain portions and facilitate comparison across different vocabularies.

for BBPE tends to be much larger when source character set and target character set have very different sizes (for example $11 \mathrm{~K}$ for $\mathrm{X}$-En source side and $0.1 \mathrm{~K}$ for the target side). And this situation becomes more severe when BBPE vocabulary size increases. In this case, alignments may be more difficult to learn during model training, since target tokens need attentions on multiple source tokens more often.

\section{Importance of Contextualization}

We compare three different ways of contextualizing token embeddings; none, 1-layer convolution and 1-layer bi-GRU, on X-En with $T_{\text {base }}$ model. We observe from Figure 4 that all kinds of vocabularies can benefit from embedding contextualization. Performance gains are more significant on finegrained vocabularies: byte, character and BBPE. For BBPE, long-range contextual information from Bi-GRU brings over $4 \%$ gain on validation BLEU in all the cases. Encoding context in the token embeddings reduces the difficulties of learning attentions on multiple source tokens and makes model training easier. In the following experiments, we contextualize BBPE with Bi-GRU by default. We denote (B)BPE with Bi-GRU as "(B)BPE $<$ size $>+$ " and the one without contextualization as "(B)BPE $<$ size $>$ ". And we similarly define "Byte+" and "Char+". 


\begin{tabular}{|c|c|c|c|c|c|c|c|c|c|c|c|c|c|c|c|c|}
\hline & \multirow{2}{*}{$\begin{array}{l}\text { Byte } \\
256\end{array}$} & \multicolumn{8}{|c|}{ BBPE } & \multicolumn{3}{|c|}{ Char } & \multicolumn{3}{|c|}{ BPE } \\
\hline & & & $1 \mathrm{~K}$ & $2 \mathrm{~K}$ & $3 \mathrm{~K}$ & $4 \mathrm{~K}$ & $8 \mathrm{~K}$ & $11 \mathrm{~K}$ & $16 \mathrm{~K}$ & $32 \mathrm{~K}$ & $3 \mathrm{~K}$ & $8 \mathrm{~K}$ & $11 \mathrm{~K}$ & $8 \mathrm{~K}$ & $16 \mathrm{~K}$ & $32 \mathrm{~K}$ \\
\hline \multirow[t]{2}{*}{ En-De } & Source & 143 & 57 & 48 & 43 & 41 & 36 & & 33 & 30 & 143 & & & 40 & 33 & 31 \\
\hline & Target & 160 & 64 & 55 & 50 & 48 & 42 & & 38 & 35 & 157 & & & 43 & 36 & 32 \\
\hline \multirow[t]{2}{*}{ Ja-En } & Source & 55 & 28 & 26 & & 24 & 23 & & 21 & 21 & & 19 & & & 12 & 10 \\
\hline & Target & 53 & 23 & 20 & & 17 & 15 & & 15 & 13 & & 52 & & & 15 & 14 \\
\hline \multirow[t]{2}{*}{ X-En } & Source & 126 & 77 & 70 & & 65 & 62 & 60 & 59 & 57 & & & 89 & & 40 & 32 \\
\hline & Target & 103 & 49 & 43 & & 37 & 33 & 32 & 30 & 27 & & & 103 & & 35 & 30 \\
\hline
\end{tabular}

Table 4: Average lengths of training sentences tokenized with different vocabularies.

\section{BBPE on Noisy Character Sets}

The En-De training set has quite a few noisy sentence pairs often containing a few non-latin alphabets due to misalignment and code-switched sentences. This leads to a $3.4 \mathrm{~K}$ character set, while in contrast, English and German both have less than 30 alphabets. Since BPE includes all characters, those rare characters will waste quite a lot of BPE vocabulary slots. For comparison, we try with small BBPE $2 \mathrm{~K}$ and $4 \mathrm{~K}$ vocabulary where rare characters are excluded. We find that their performance are comparable to the BPE $32 \mathrm{~K}$ baseline while having smaller model capacity (see table 5).

\begin{tabular}{cl|c|c} 
& & Test BLEU & Params \\
\hline$T_{\text {base }}$ & Byte+ & 26.59 & $45 \mathrm{M}$ \\
& BBPE 2K+ & 26.98 & $47 \mathrm{M}$ \\
& BBPE 4K+ & 27.08 & $47 \mathrm{M}$ \\
& Char+ & 26.73 & $47 \mathrm{M}$ \\
& BPE 32K & 27.31 & $61 \mathrm{M}$ \\
& BPE 32K+ & $\mathbf{2 7 . 4 1}$ & $62 \mathrm{M}$ \\
& BPE 37K ${ }^{\star}$ & 27.3 & $65 \mathrm{M}$ \\
\hline$T_{\text {big }}$ & Byte+ & 26.94 & $181 \mathrm{M}$ \\
& BBPE 2K+ & $\mathbf{2 8 . 7 8}$ & $183 \mathrm{M}$ \\
& BBPE 4K+ & 28.27 & $185 \mathrm{M}$ \\
& Char+ & 27.24 & $185 \mathrm{M}$ \\
& BPE 32K & 28.36 & $210 \mathrm{M}$ \\
& BPE 32K+ & 28.77 & $215 \mathrm{M}$ \\
& BPE 37K ${ }^{\star}$ & 28.4 & $213 \mathrm{M}$
\end{tabular}

Table 5: En-De test BLEU. $\star$ (Vaswani et al. 2017).

\section{BBPE on Character-Rich Languages}

Languages using logographic writing systems, such as Chinese and Japanese, can have over $50 \mathrm{~K}$ characters, while only a small portion of them are frequently used. Our Ja-En dataset has a set of $7.9 \mathrm{~K}$ characters, where $99.99 \%$ tokens in the training set are covered by the top $2.4 \mathrm{~K}$ characters. With this observation, we experiment with BBPE $4 \mathrm{~K}$ which is roughly $50 \%$ of the character set size. We find that BBPE is comparable to BPE and even outperforms BPE when using larger $T_{b i g}$ model (see table 6).

\section{BBPE on Many-to-En Translation}

Our many-to-En dataset contains 58 languages (parallelly to English) and $10.8 \mathrm{~K}$ characters from different writing sys-

\begin{tabular}{|c|c|c|c|c|c|}
\hline & 71 & TED & JES & All \\
\hline \multicolumn{2}{|c|}{$\begin{array}{l}\text { \# of train samples } \\
\text { \# of test samples }\end{array}$} & $\begin{array}{l}440 \mathrm{~K} \\
1.2 \mathrm{~K}\end{array}$ & $\begin{array}{c}223 \mathrm{~K} \\
8.5 \mathrm{~K}\end{array}$ & $\begin{array}{c}2.8 \mathrm{M} \\
2 \mathrm{~K}\end{array}$ & $\begin{array}{c}3.5 \mathrm{M} \\
11.7 \mathrm{~K}\end{array}$ \\
\hline \multicolumn{2}{|c|}{ Michel et.al. (2018) } & 20.77 & 13.25 & 18.00 & - \\
\hline$T_{\text {base }}$ & & & & $\begin{array}{l}15.69 \\
16.10 \\
15.68 \\
\mathbf{1 6 . 1 8}\end{array}$ & $\begin{array}{l}16.27 \\
16.80 \\
16.43 \\
\mathbf{1 7 . 1 9}\end{array}$ \\
\hline & $\begin{array}{l}\text { BBPE 4K+ } \\
\text { BPE } 16 \mathrm{~K}+\end{array}$ & $\begin{array}{l}23.68 \\
23.88 \\
23.71 \\
\mathbf{2 4 . 0 8}\end{array}$ & $\begin{array}{c}16.08 \\
\mathbf{1 9 . 0} \\
16.69 \\
18.34\end{array}$ & $\begin{array}{l}16.29 \\
\mathbf{1 7 . 9 3} \\
17.01 \\
17.89\end{array}$ & $\begin{array}{l}17.46 \\
\mathbf{1 9 . 5 8} \\
18.33 \\
19.14\end{array}$ \\
\hline
\end{tabular}

Table 6: Ja-En test BLEU scores.

tems, between which characters are not necessarily shared. The characters, however, share byte $n$-grams. We experiment with BBPE $2 \mathrm{~K}$ and $4 \mathrm{~K}$ that have $12.5 \%$ and $25 \%$ size of the baseline BPE vocabulary. As shown in Table 7, both of them beat the BPE baseline on overall BLEU as well as on most of the languages both with high and low resources (Note that the test set is as large as $165 \mathrm{~K}$ and even small gaps in BLEU may suggest significant difference). We also notice that byte model and character model perform significantly better than all BPE and BBPE models in this multilingual setting. This might be because that BBPE and BPE suffer from imbalanced source and target sentence lengths as well as various token granularities in multilingual parallel sentences (sources in different languages and granularities into same targets). Nonetheless, BBPE is still the most practical solution since it makes a good balance between performance (better BLEU than BPE) and speed (much shorter tokenized sentences than characters and bytes).

\section{Transfer Learning on Unseen Characters}

Because BBPE contains all UTF- 8 bytes and has no outof-vocabulary tokens, BBPE-based models can be transferred between languages with non-overlapping character sets. In comparison, it is impossible to do so with characterbased vocabularies without replacing the vocabulary and retraining embeddings from scratch. Our Si-En dataset has 77 Sinhala scripts that are disjoint with the X-En character set. We experiment transferring a pretrained (on X-En) BBPE $4 \mathrm{~K} T_{\text {flores }}$ model to this dataset while reusing the original 


\begin{tabular}{|c|c|c|c|c|c|c|c|c|c|c|c|}
\hline & $\mathrm{Ar}$ & De & $\mathrm{He}$ & It & $\mathrm{Az}$ & $\mathrm{Be}$ & Gl & Sk & All & Params \\
\hline \multirow{2}{*}{\multicolumn{2}{|c|}{$\begin{array}{l}\text { \# of train examples } \\
\text { \# of test examples }\end{array}$}} & $213 \mathrm{~K}$ & $167 \mathrm{~K}$ & $211 \mathrm{~K}$ & $203 K$ & $5.9 \mathrm{~K}$ & $4.5 \mathrm{~K}$ & $10 \mathrm{~K}$ & $61 \mathrm{~K}$ & $5.1 \mathrm{M}$ & \\
\hline & & $6 \mathrm{~K}$ & $4.5 \mathrm{~K}$ & $5.5 \mathrm{~K}$ & $5.6 \mathrm{~K}$ & $0.9 \mathrm{~K}$ & $0.7 \mathrm{~K}$ & $1 \mathrm{~K}$ & $2.4 \mathrm{~K}$ & $165 \mathrm{~K}$ & \\
\hline \multicolumn{2}{|c|}{$\begin{array}{l}\text { Aharoni et al. } 19 \\
\text { Neubig \& Hu } 18\end{array}$} & 25.93 & 28.87 & 30.19 & 32.42 & 11.7 & 18.3 & 29.1 & 28.3 & & \\
\hline \multirow{2}{*}{$T_{\text {base }}$} & Byte+ & 31.13 & 35.98 & 36.77 & 38.36 & 14.64 & 25.12 & 35.12 & 33.08 & 30.38 & $45 \mathrm{M}$ \\
\hline & Chart & 31.52 & 36.73 & 36.85 & 38.62 & 15.40 & 24.90 & 35.44 & 33.31 & 30.75 & $51 \mathrm{M}$ \\
\hline \multirow[t]{6}{*}{$T_{\text {base }}$} & BBPE $2 \mathrm{~K}+$ & 30.79 & 35.53 & 36.27 & 37.82 & 13.64 & 24.70 & 34.17 & 32.83 & 29.91 & $46 \mathrm{M}$ \\
\hline & BBPE $4 \mathrm{~K}+$ & 30.64 & 34.93 & 36.07 & 37.62 & 13.76 & 24.84 & 33.90 & 32.12 & 29.74 & $47 \mathrm{M}$ \\
\hline & BPE $16 \mathrm{~K}$ & 29.70 & 34.35 & 34.47 & 37.02 & 13.28 & 24.61 & 33.55 & 31.72 & 29.00 & $53 \mathrm{M}$ \\
\hline & BPE $16 \mathrm{~K}+$ & 30.20 & 34.97 & 35.55 & 37.49 & 12.65 & 23.66 & 33.95 & 32.16 & 29.62 & $54 \mathrm{M}$ \\
\hline & BPE $32 \mathrm{~K}$ & 29.02 & 34.08 & 34.18 & 36.63 & 12.56 & 22.48 & 32.33 & 31.26 & 28.81 & $61 \mathrm{M}$ \\
\hline & BPE $32 \mathrm{~K}+$ & 29.87 & 34.64 & 35.26 & 37.43 & 12.35 & 22.05 & 33.62 & 31.61 & 29.43 & $62 \mathrm{M}$ \\
\hline
\end{tabular}

Table 7: X-En test BLEU on all 58 languages, top-4 (Ar, De, He, It) and bottom-4 (Az, Be, Gl, Sk) languages by number of training samples. Note that the test set is very large $(165 \mathrm{~K})$ and even small gaps in BLEU may suggest significant difference.

\begin{tabular}{cc|cc|c} 
& & Train & Finetune & BLEU \\
\hline$T_{\text {flores }}$ & BPE 5K & Si-En & - & 7.2 \\
& BBPE 4K+ & Si-En & - & 7.1 \\
\hline$T_{\text {flores }}$ & BBPE 4K+ & X-En & - & 0.3 \\
& BBPE 4K+ & X-En & enc & 8.3 \\
& BBPE 4K+ & X-En & enc, dec & 8.1 \\
& BBPE 4K+ & X-En & embed, enc & $\mathbf{9 . 0}$ \\
& BBPE 4K+ & X-En & all & 8.6
\end{tabular}

Table 8: Transferring pretrained X-En model to Si-En. BBPE 4K is learned on X-En. * (Guzmán et al. 2019).

vocabulary. As shown in table 8 , the transferred model gains 0.9-1.8 BLEU points compared to the baselines, suggesting the generality of pretrained BBPE embeddings and its ability to adapt to different languages with unseen characters. This transfer learning paradigm is free from the limitation of outof-vocabulary tokens and can be very generic. We just show the extreme case of totally unseen character set, but the pretrained model may also be transferred to any languages and datasets to improve performance or warm-start model training to save time.

\section{Related Work}

Subword Vocabularies Previous works have shown that finer-grained vocabularies consistently outperforms wordlevel vocabularies in many settings, for example, vocabularies based on morpheme segmentation (Nießen and Ney 2000; Luong, Socher, and Manning 2013), byte-pair encoding (Sennrich, Haddow, and Birch 2015) and vocabularies from unigram language model (Kudo 2018). Our bytelevel subword vocabularies are based on byte-pair encoding, while we use bytes as the basic units to compose subwords.

Character Vocabulary Existing works also explored pure character vocabulary for machine translation. (Kim et al. 2016) proposed building word representations from character ones; (Chung, Cho, and Bengio 2016) removed the re- striction of word boundaries and directly learned decoding in character level; (Lee, Cho, and Hofmann 2017) further extended it to a fully character-level model in a multilingual setting; (Cherry et al. 2018) showed that character-level models generally outperforms subword-level ones given enough model capacity.

Byte-Level Vocabularies The closest work to ours is the byte-level BPE vocabulary used in GPT-2, a large-scale English language model (Radford et al. 2019). They however rely heavily on hard-coded merging rules and have not conducted any analysis on how their bye-level BPE impacts the quality of language modeling. A vocabulary consisting purely of bytes has previously been used in several natural language processing tasks: part-of-speech tagging and named entity recognition (Gillick et al. 2016), translation(Costa-Jussà, Escolano, and Fonollosa 2017), machine reading (Kenter, Jones, and Hewlett 2018) and speech recognition (Li et al. 2019).

Transformer with Convolution or RNN There are evidences for performance gains from combining Transformer with convolutional or recurrent layers in the area of NMT (Chen et al. 2018), speech recognition (Li et al. 2019; Mohamed, Okhonko, and Zettlemoyer 2019) and language modeling (Chenguang Wang 2019).

\section{Conclusion}

We proposed BBPE which builds a byte-level subword vocabulary for machine translation. It results in a much more compact vocabulary than character-based ones do without the loss of performance. In multilingual settings, the former often outperforms the latter. BBPE does not have any outof-vocabulary tokens, allowing us to transfer a model using BBPE between languages with non-overlapping vocabularies. This transfer learning paradigm is actually very generic and can be applied to any languages and datasets for performance gain or training acceleration. With the same vocabulary size, BBPE segments sentences into shorter sequences 


\begin{tabular}{|c|c|c|c|}
\hline Original & & 質問して_証明と証拠を求めましょう & Ask_questions,_demand__proof,_demand_evidence. \\
\hline \multirow[t]{3}{*}{ Byte } & & $\begin{array}{l}\text { E8 B3 AA E5 } 95 \text { 8F E3 } 8197 \text { E3 } 81 \text { A6 E2 } 9681 \text { E8 A8 BC E6 } 98 \text { 8E } \\
\text { E3 } 81 \text { A8 E8 A8 BC E6 8B A0 E3 } 8292 \text { E6 B1 } 82 \text { E3 } 8281 \text { E3 } 81 \text { BE } \\
\text { E3 } 8197 \text { E3 } 8287 \text { E3 } 8186\end{array}$ & $\begin{array}{l}41736 \mathrm{~B} \text { E2 } 9681717565737469 \text { 6F } 6 \mathrm{E} 732 \mathrm{C} \text { E2 } 96816465 \text { 6D } \\
616 \mathrm{E} 64 \mathrm{E} 2968170726 \mathrm{~F} 6 \mathrm{~F} 66 \text { 2C E2 } 968164656 \mathrm{D} 616 \mathrm{E} 64 \mathrm{E} 2 \\
968165766964656 \mathrm{E} 6365 \text { 2E }\end{array}$ \\
\hline & $1 \mathrm{~K}$ & $\begin{array}{l}\text { E8 B3 AA E595 8F しE381 A6 _E8 A8 BC 明 E381 A8 E8 A8 BC E6 } \\
\text { 8B A0 をE6 B1 } 82 \text { めE381 BE しょう }\end{array}$ & A s k_quest ions, _ dem and _ pro of , _ dem and _ev idence. \\
\hline & $2 \mathrm{~K}$ & $\begin{array}{l}\text { E8 B3 AA 問 しE381 A6_E8 A8BC 明 E381 A8 E8 A8BC E68B A0 を } \\
\text { E6 B1 } 82 \text { めE381 BE しょう }\end{array}$ & A sk__qu est ion s, _ d em and _ pro o f, _ d em and _ e vid ence. \\
\hline \multirow[t]{4}{*}{ BBPE } & $4 \mathrm{~K}$ & $\begin{array}{l}\text { E8 B3 AA 問 しE381 A6 _E8 A8BC 明E381 A8 E8 A8BC 拠をE6 B1 } \\
82 \text { めE381 BE しょう }\end{array}$ & 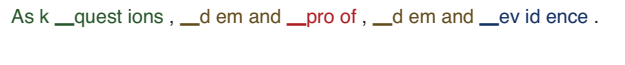 \\
\hline & $8 \mathrm{~K}$ & $\begin{array}{l}\text { E8 B3 AA問 しE381 A6 _E8 A8BC 明E381 A8 E8 A8BC 拠をE6 B1 } \\
\text { 82めE381 BE しょう }\end{array}$ & As k_questions, _demand_pro of , _ demand__evidence . \\
\hline & $16 \mathrm{~K}$ & $\begin{array}{l}\text { E8 B3 AA問 しE381 A6_E8 A8BC 明E381 A8 E8 A8BC 拠 をE6 B1 } \\
\text { 82めE381 BE しょう }\end{array}$ & As k_questions, _demand__proof, __demand__evidence . \\
\hline & $32 \mathrm{~K}$ & $\begin{array}{l}\text { E8 B3 AA問しE381 A6 _E8 A8BC 明E381 A8 E8 A8BC 拠 をE6 B1 } 82 \\
\text { めE381 BE しょう }\end{array}$ & As k_questions, _ demand__proof, _ demand__evidence . \\
\hline CHAR & & 質問してー証明と証拠を求めましょう & $\begin{array}{l}\text { Ask_questions, }, \text { demand } \_ \text {proof, }, \text { demand } \\
\text { evidence. }\end{array}$ \\
\hline \multirow[t]{2}{*}{ BPE } & $16 \mathrm{~K}$ & 質問 して—証明と証拠 を求めましょう & As k_questions, _demand__pro of , _ demand__evidence. \\
\hline & $32 \mathrm{~K}$ & 質問 して—証明 と 証拠 を求めましょう & As k_questions, _demand__proof, _ demand__evidence . \\
\hline
\end{tabular}

Figure 5: An example from Ja-En tokenized with different vocabularies. Raw spaces are replaced by underscores and spaces are used to split tokens. We can observe how tokens look like as the tokenization granularity goes from fine to coarse: Byte (256) $\rightarrow$ BBPE $(1 \mathrm{~K}, 2 \mathrm{~K}, 4 \mathrm{~K}, 8 \mathrm{~K}) \rightarrow$ Char $(8 \mathrm{~K}) \rightarrow \mathrm{BBPE}(16 \mathrm{~K}, 32 \mathrm{~K}) \rightarrow \mathrm{BPE}(16 \mathrm{~K}, 32 \mathrm{~K})$.

than character-based methods do, leading to faster training and inference. Our future work includes: eliminating sourcetarget sentence length imbalance; evaluating BBPE in oneto-many and many-to-many translation settings; exploring better segmentation algorithms for byte-level subwords.

\section{References}

Bahdanau, D.; Cho, K.; and Bengio, Y. 2014. Neural machine translation by jointly learning to align and translate. arXiv preprint arXiv:1409.0473.

Cettolo, M.; Girardi, C.; and Federico, M. 2012. Wit3: Web inventory of transcribed and translated talks. In Conference of European Association for Machine Translation, 261-268. Chen, M. X.; Firat, O.; Bapna, A.; Johnson, M.; Macherey, W.; Foster, G.; Jones, L.; Parmar, N.; Schuster, M.; Chen, Z.; et al. 2018. The best of both worlds: Combining recent advances in neural machine translation. arXiv preprint arXiv:1804.09849.

Chenguang Wang, Mu Li, A. J. S. 2019. Language models with transformers. In ArXiv e-prints.

Cherry, C.; Foster, G.; Bapna, A.; Firat, O.; and Macherey, W. 2018. Revisiting character-based neural machine translation with capacity and compression. arXiv preprint arXiv:1808.09943.

Cho, K.; van Merrienboer, B.; Gulcehre, C.; Bahdanau, D.; Bougares, F.; Schwenk, H.; and Bengio, Y. 2014. Learning phrase representations using rnn encoder-decoder for statistical machine translation. In Proceedings of the 2014 Con- ference on Empirical Methods in Natural Language Processing (EMNLP), 1724-1734.

Chung, J.; Cho, K.; and Bengio, Y. 2016. A character-level decoder without explicit segmentation for neural machine translation. arXiv preprint arXiv:1603.06147.

Costa-Jussà, M. R.; Escolano, C.; and Fonollosa, J. A. 2017. Byte-based neural machine translation. In Proceedings of the First Workshop on Subword and Character Level Models in NLP.

Gillick, D.; Brunk, C.; Vinyals, O.; and Subramanya, A. 2016. Multilingual language processing from bytes. In Proceedings of NAACL-HLT.

Guzmán, F.; Chen, P.-J.; Ott, M.; Pino, J.; Lample, G.; Koehn, P.; Chaudhary, V.; and Ranzato, M. 2019. Two new evaluation datasets for low-resource machine translation: Nepali-english and sinhala-english.

Kaiser, L.; Gomez, A. N.; and Chollet, F. 2017. Depthwise separable convolutions for neural machine translation. arXiv preprint arXiv:1706.03059.

Kenter, T.; Jones, L.; and Hewlett, D. 2018. Byte-level machine reading across morphologically varied languages. In Thirty-Second AAAI Conference on Artificial Intelligence.

Kim, Y.; Jernite, Y.; Sontag, D.; and Rush, A. M. 2016. Character-aware neural language models. In Thirtieth AAAI Conference on Artificial Intelligence.

Kudo, T., and Richardson, J. 2018. Sentencepiece: A simple and language independent subword tokenizer and deto- 
kenizer for neural text processing. In Proceedings of the 2018 Conference on Empirical Methods in Natural Language Processing: System Demonstrations, 66-71.

Kudo, T. 2018. Subword regularization: Improving neural network translation models with multiple subword candidates. In Proceedings of the 56th Annual Meeting of the Association for Computational Linguistics (Volume 1: Long Papers), 66-75.

Lee, J.; Cho, K.; and Hofmann, T. 2017. Fully characterlevel neural machine translation without explicit segmentation. Transactions of the Association for Computational Linguistics 5:365-378.

Li, B.; Zhang, Y.; Sainath, T.; Wu, Y.; and Chan, W. 2019. Bytes are all you need: End-to-end multilingual speech recognition and synthesis with bytes. In 2019 IEEE International Conference on Acoustics, Speech and Signal Processing (ICASSP).

Luong, T.; Socher, R.; and Manning, C. 2013. Better word representations with recursive neural networks for morphology. In Proceedings of the Seventeenth Conference on Computational Natural Language Learning, 104-113.

Michel, P., and Neubig, G. 2018. Mtnt: A testbed for machine translation of noisy text. arXiv preprint arXiv:1809.00388.

Mohamed, A.; Okhonko, D.; and Zettlemoyer, L. 2019. Transformers with convolutional context for asr. arXiv preprint arXiv:1904.11660.

Neubig, G. 2011. The Kyoto free translation task. http://www.phontron.com/kftt.

Nießen, S., and Ney, H. 2000. Improving smt quality with morpho-syntactic analysis. In Proceedings of the 18th conference on Computational linguistics-Volume 2, 1081-1085. Association for Computational Linguistics.

Ott, M.; Edunov, S.; Baevski, A.; Fan, A.; Gross, S.; Ng, N.; Grangier, D.; and Auli, M. 2019. fairseq: A fast, extensible toolkit for sequence modeling. In Proceedings of NAACLHLT 2019: Demonstrations.

Papineni, K.; Roukos, S.; Ward, T.; and Zhu, W.-J. 2002. Bleu: a method for automatic evaluation of machine translation. In Proceedings of the 40th annual meeting on association for computational linguistics, 311-318. Association for Computational Linguistics.

Post, M. 2018. A call for clarity in reporting BLEU scores. In Proceedings of the Third Conference on Machine Translation: Research Papers, 186-191. Association for Computational Linguistics.

Pryzant, R.; Chung, Y.; Jurafsky, D.; and Britz, D. 2017. JESC: Japanese-English Subtitle Corpus. ArXiv e-prints.

Radford, A.; Wu, J.; Child, R.; Luan, D.; Amodei, D.; and Sutskever, I. 2019. Language models are unsupervised multitask learners.

Sennrich, R.; Haddow, B.; and Birch, A. 2015. Neural machine translation of rare words with subword units. In Proceedings of the 54th Annual Meeting of the Association for Computational Linguistics.
Sutskever, I.; Vinyals, O.; and Le, Q. V. 2014. Sequence to sequence learning with neural networks. In Advances in neural information processing systems, 3104-3112.

Vaswani, A.; Shazeer, N.; Parmar, N.; Uszkoreit, J.; Jones, L.; Gomez, A. N.; Kaiser, L.; and Polosukhin, I. 2017. Attention is all you need. In Advances in Neural Information Processing Systems, 5998-6008.

Ye, Q.; Devendra, S.; Matthieu, F.; Sarguna, P.; and Graham, N. 2018. When and why are pre-trained word embeddings useful for neural machine translation. In HLT-NAACL. 